\title{
BMJ Open Improving smoking cessation care in pregnancy at Aboriginal Medical Services: 'ICAN QUIT in Pregnancy' step-wedge cluster randomised study
}

\author{
Yael Bar-Zeev, ${ }^{\circ}$ Michelle Bovill, ${ }^{1}$ Billie Bonevski, ${ }^{1}$ Maree Gruppetta, ${ }^{2}$ \\ Christopher Oldmeadow, ${ }^{3}$ Kerrin Palazzi, ${ }^{3}$ Louise Atkins, ${ }^{4}$ Jennifer Reath, ${ }^{5}$ \\ Gillian Sandra Gould, ${ }^{1}$ the 'ICAN QUIT in Pregnancy' Pilot Group
}

To cite: Bar-Zeev Y, Bovill M, Bonevski B, et al. Improving smoking cessation care in pregnancy at Aboriginal Medical Services: 'ICAN QUIT in Pregnancy' step-wedge cluster randomised study. BMJ Open 2019:9:e025293. doi:10.1136/ bmjopen-2018-025293

- Prepublication history and additional material for this paper are available online. To view, please visit the journal online (http://dx.doi.org/10.1136/ bmjopen-2018-025293).

Received 10 July 2018 Revised 24 April 2019 Accepted 26 April 2019
Check for updates

(C) Author(s) (or their employer(s)) 2019. Re-use permitted under CC BY-NC. No commercial re-use. See rights and permissions. Published by BMJ.

For numbered affiliations see end of article.

Correspondence to Dr Yael Bar-Zeev; yael.barzeev@uon.edu.au

\section{ABSTRACT}

Objectives This study aimed to examine the impact of the 'ICAN QUIT in Pregnancy' intervention on individual health providers (HPs) smoking cessation care (SCC) knowledge, attitudes and practices in general, and specifically regarding nicotine replacement therapy (NRT) prescription. Design Step-wedge clustered randomised controlled study. HPs answered a preintervention and 1-6 months postintervention survey.

Setting Six Aboriginal Medical Services (AMSs) in three states of Australia.

Participants All HPs were invited to participate. Of 93 eligible, 50 consented (54\%), 45 completed the presurvey (90\%) and 20 the post (40\%).

Intervention Included three 1-hour webinar sessions, educational resource package and free oral NRT.

Outcomes HPs knowledge was measured using two composite scores-one from all 24 true/false statements, and one from 12 NRT-specific statements. Self-assessment of 22 attitudes to providing SCC were measured using a five-point Likert scale (Strongly disagree to Strongly agree). Two composite mean scores were calculated-one for 15 general SCC attitudes, and one for 7 NRT-specific attitudes. Self-reported provision of SCC components was measured on a five-point Likert scale (Never to Always). Feasibility outcomes, and data collected on the service and patient level are reported elsewhere. Results Mean knowledge composite scores improved from pre to post $(78 \%$ vs $84 \%$ correct, difference 5.95 , $95 \% \mathrm{Cl} 1.57$ to 10.32). Mean NRT-specific knowledge composite score also improved (68\% vs $79 \%$ correct, difference $9.9,95 \% \mathrm{Cl} 3.66$ to 16.14$)$. Mean attitude composite score improved (3.65 (SD 0.4) to 3.87 (SD 0.4), difference $0.23,95 \% \mathrm{Cl} 0.05$ to 0.41 ). Mean NRT-specific attitudes composite score also improved (3.37 (SD 0.6) to 3.64 (SD 0.7), difference $0.36,95 \% \mathrm{Cl} 0.13$ to 0.6 ). Selfreported practices were unchanged, including prescribing NRT.

Conclusions A multicomponent culturally sensitive intervention in AMSs was feasible, and might improve HPs provision of SCC to pregnant Aboriginal women. Changes in NRT prescription rates may require additional intensive measures.

Trial registration number ACTRN 12616001603404; Results.
Strengths and limitations of this study

- The first study in Australia that specifically targeted health providers' (HPs) care for pregnant Aboriginal and Torres Strait Islander women who smoke.

- The first intervention that had a major focus on improving prescription rates of nicotine replacement therapy to support smoking cessation during pregnancy.

- The intervention, including the novel use of live interactive webinar training, was informed by theory and based on a solid foundation of prior formative research.

- This study was a pilot aimed to assess feasibility, and was not powered to assess the effectiveness of the intervention.

- This study included HPs who work at Aboriginal Medical Services only, and may not be generalised to other antenatal care settings that Aboriginal and Torres Strait Islander women might attend.

\section{INTRODUCTION}

Smoking during pregnancy remains a serious public health problem. Global rates range from $0.2 \%$ to $38 \%$, with higher rates of $30 \%-50 \%$, across high-priority subpopulations. ${ }^{12}$ In Australia, $10 \%$ of pregnant women smoke, with the highest rates across socially disadvantaged subpopulations, such as Aboriginal and Torres Strait Islander women (44\%) (hereafter referred to as 'Aboriginal' women with acknowledgement of the distinct cultures). ${ }^{3}$ The Aboriginal population compromise $3 \%$ of the total Australian population, ${ }^{4}$ but due to historical, cultural and social reasons, have the highest smoking prevalence $(42 \%) .^{56}$ Tobacco was introduced to Aboriginal people through trade with Indonesian fisherman and quickly became embedded in the social and ceremonial life. ${ }^{7}$ Colonisation had a huge impact on smoking rates as tobacco was often used by 
European settlers as an accepted method of payment to Aboriginal people. ${ }^{8}$

Reducing smoking prevalence during pregnancy can lead to significant positive impacts on long-term development of Aboriginal babies and help to reduce the health gap between the Non-Aboriginal and the Aboriginal population. ${ }^{9}$ Reducing tobacco use is considered to be one of the key areas for intervention to improve infant and maternal health outcomes, under the 2018 'Closing the Gap' governmental strategy for Aboriginal Australians. ${ }^{10}$

Pregnant Aboriginal women face many barriers to quitting smoking, ${ }^{11-13}$ including multiple and intercurrent life stressors, and living in communities with high smoking rates. A significant barrier, commonly cited, is the lack of adequate support from health providers (HPs), with inconsistent health messages often provided. ${ }^{13-15}$

Effective interventions to increase rates of smoking cessation during pregnancy include behavioural counselling, incentives and feedback (ie, when the pregnant woman is offered an objective measurement of the effects of tobacco smoking, such as the level of carbon monoxide in expired air). ${ }^{1}$

The most recent Cochrane review on using pharmacotherapy to aid smoking cessation in pregnancy concluded that nicotine replacement therapy (NRT) increased cessation rates by $40 \% .{ }^{16}$ On average, quit rates increased from $9.2 \%$ with placebo to $12.2 \%$ with NRT. ${ }^{16}$ However, excluding non-placebo controlled studies resulted in non-significant changes. Therefore, the evidence regarding the use of NRT in pregnancy is still not robust. Furthermore, adherence to NRT was identified as a problem. ${ }^{16}$

Australian smoking cessation guidelines recommend the use of NRT if a pregnant woman has not been able to quit smoking with counselling alone. ${ }^{17}$ Despite this, $25 \%$ of Australian general practitioners and obstetricians have reported they would 'never' prescribe NRT during pregnancy, with 55\% reporting safety concerns about NRT use in pregnancy. ${ }^{18}$ A recent systematic review identified that HPs are not providing pregnant women adequate support to quit, specifically in regard to behavioural counselling, referral to specialist support (such as the Quitline) and prescription of NRT. ${ }^{19}$ Several reviews have outlined numerous barriers HPs face to providing smoking cessation care (SCC) during pregnancy, including lack of knowledge and skills, low confidence in ability to counsel, low optimism regarding the effectiveness of treatment, and lack of time and resources. ${ }^{20}{ }^{21}$ The same barriers were echoed recently in two Australian studies including HPs treating Aboriginal pregnant mothers. ${ }^{22} 23$

To date, there have been two published studies focusing on smoking cessation in Australian Aboriginal pregnant women, ${ }^{24}{ }^{25}$ neither focused on the HPs provision of care, or on the use of NRT during pregnancy. Both implemented face-to-face smoking cessation training of HPs as part of the intervention. Despite an increase in webinar use as a mechanism for training HPs, very few studies have tested its effectiveness in regard to smoking cessation training. ${ }^{26}{ }^{27}$ A recent systematic review on interventions to improve HPs provision of SCC during pregnancy identified 14 different interventions, none of which included webinar training, and none included components aimed specifically at improving NRT prescription. ${ }^{28}$

The Indigenous Counselling and Nicotine (ICAN) QUIT in Pregnancy trial ${ }^{29}$ was a pilot study with an overall aim of testing the feasibility and acceptability of a multicomponent smoking cessation training intervention for HPs in Aboriginal Medical Services (AMSs). The intervention included 3 hours of live interactive webinar training, an educational resource package and free oral NRT. ${ }^{29}$ The intervention was developed collaboratively with two AMSs and a Stakeholder and Consumer Aboriginal Advisory Panel guiding the development and implementation of the intervention to ensure Aboriginal community ownership and cultural sensitivity. A full description of the development of this intervention is provided elsewhere. ${ }^{30}$

The results of this pilot study will inform the development and design of a larger cluster randomised controlled trial (SISTAQUIT-Supporting Indigenous Smokers To Assist Quitting).

This study examined the impact of 'ICAN QUIT in Pregnancy' on HPs' knowledge, attitudes and practices regarding SCC during pregnancy in general, and specifically on recommending NRT during pregnancy. The usefulness of the educational resources was also assessed.

\section{METHODS \\ Design}

The 'ICAN QUIT in Pregnancy' study was a step-wedge clustered randomised trial (figure 1). Full details of the study can be found in the protocol manuscript (online supplementary file 1). ${ }^{29}$ In short, six AMSs were randomised into three clusters, with the intervention sequentially delivered to each cluster 2 months following study commencement, staggered by 1 month. The trial included data collection at the service and HPs levels, and from pregnant participants with the primary outcomes focused on feasibility. ${ }^{29}$ In this manuscript, we report on the HPs secondary outcome data. The feasibility outcomes, service level and women's level data are reported elsewhere ${ }^{31}$ (Bovill et al, Wingadhan Birrang (woman's journey) of smoking cessation during pregnancy: Aboriginal and Torres Strait Islander women participating in the Indigenous Counselling and Nicotine (ICAN) QUIT in Pregnancy pilot study). All HPs in each service were invited to receive the training, and complete a survey pretraining and post-training (figure 1). Originally, HPs were to complete two postsurveys-at 1 and 6 months post-training. Few HPs completed the postsurvey 1-month post-training, and multiple reminders to complete the postsurvey were required over the follow-up period. Therefore, only one postsurvey was collected from each HP (between 1 and 6 months post-training). 

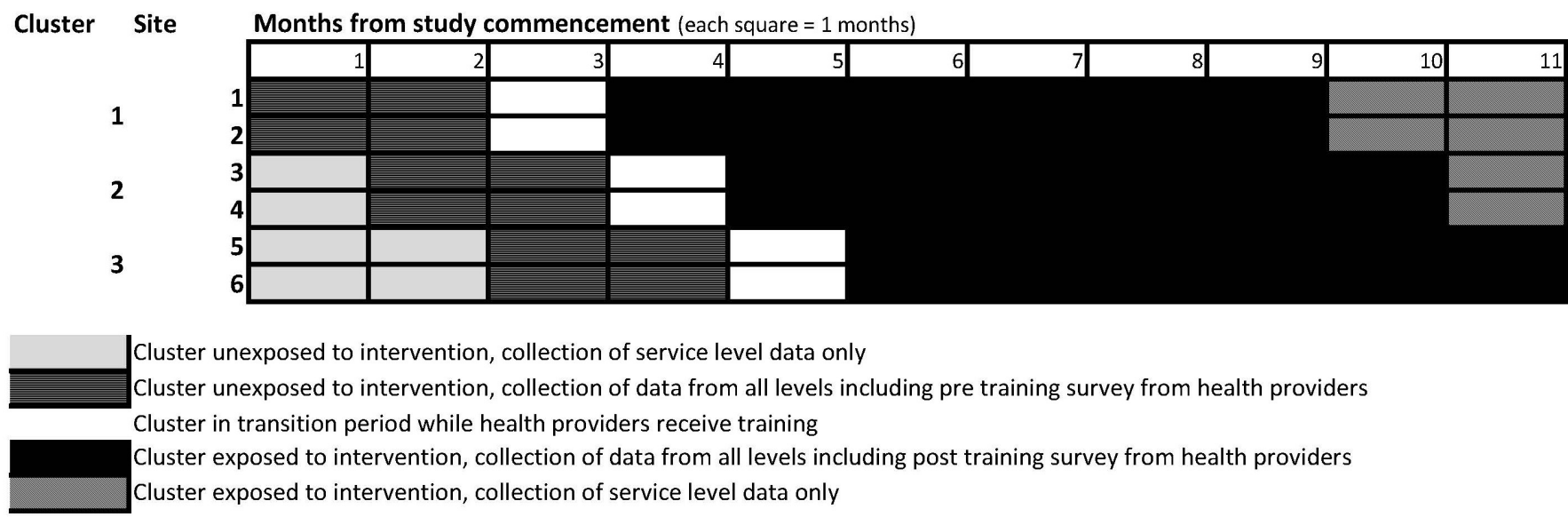

Figure 1 Schematic illustration of the step-wedge cluster study for the Indigenous Counselling and Nicotine (ICAN) QUIT in Pregnancy pilot study.

\section{Setting}

Six AMSs-one urban and five regional-in three states of Australia-New South Wales, Queensland and South Australia. The AMSs were all Aboriginal Community Controlled Health Services, which are non-government organisations operated by local Aboriginal and Torres Strait Islander communities, to deliver holistic, comprehensive and culturally appropriate healthcare to the communities that govern them through an elected board of management. ${ }^{32}$

\section{Participants}

All of the HPs from each service were invited to participate, as long as they consulted with pregnant women either for confirmation of pregnancy, antenatal care and/or routine care. These included general practitioners, midwives, Aboriginal Health Workers and other allied HPs. Aboriginal Health Workers are Aboriginal people who have undertaken specific training to work within primary care, either in non-clinical liaison or clinical roles. ${ }^{33}$ Managers were also welcomed to attend the training as non-participants.

\section{Randomisation}

Randomisation occurred at the service level only, with allocation of the AMSs to the clusters based on geographical convenience. For each cluster, the period of treatment crossover was randomised using simple randomisation. Due to the step-wedge cluster design, HPs from each service received the training, and completed the surveys at different time points in the study, staggered by 1 month between each cluster (figure 1).

\section{Intervention}

Intervention included (a) three 1-hour sessions of live interactive webinar training for HPs, (b) an educational resource package for both HPs and pregnant women and (c) free oral forms of NRT to be dispensed to women on-site. 2934 a. Webinar training was chosen to accommodate time and location constraints, important for future potential scaling of the intervention to other regional and remote AMSs. Webinars included PowerPoint presentations, embedded short videos and group discussions. ${ }^{34}$ A full description of the webinar content can be found in online supplementary file 2 . The webinars were recorded and supplied to each service. This enabled training for those HPs that could not attend the live webinar, and allowed HPs to view the webinar again if needed. Originally, the webinars were planned to be weekly, over 3 weeks, but all the services requested them to be in one 3-hour time slot, due to HPs time limits.

b. The educational resource package included resources specifically for the HPs (a treatment manual, and a mousepad depicting the NRT treatment algorithm to act as a prompt), resources specifically for the women (patient booklet, posters showing different NRT options and the differences in chemical content between NRT and a cigarette) and resources for a joint discussion (a flipchart). Development and assessment of these resources, and full details regarding the intervention can be found elsewhere. ${ }^{29} 3435$ The educational resource package was provided to the services both in hard copies (one for each HP) and in a digital copy.

c. All forms of oral NRT were supplied to the services (gum, lozenge, spray and inhalator) to be provided as needed free of charge to the women. Originally, oral NRT was to be supplied through a pharmacy voucher system, but all of the services requested that it would be dispensed directly at the service, to accommodate time and travel constraints for their patients. Due to the higher metabolism in pregnancy, ${ }^{36}$ it was advised to initiate treatment with the higher oral NRT dose (such as the $4 \mathrm{mg}$ gum or lozenge). Services were supplied with sample packs for each woman, and supplies to last for a 3 months treatment period. NRT patches were 
available for free under the Australian Government Pharmaceutical Benefits Scheme (PBS) ${ }^{37}$ In Australia, NRT can be bought without a prescription at pharmacies, but in order to qualify for the PBS subsidisation, a script from a medical doctor is required. ${ }^{37}$

The original plan was to include audit and feedback through a collection of monthly de-identified computerised data from each service regarding NRT prescription rates to pregnant women, providing each service with a monthly report on their prescription rates, compared with the other services in the study. ${ }^{29}$ Unfortunately, audit and feedback was not feasible as services took a few months to organise the computerised data, and only four services were able to provide the data regarding NRT prescriptions to pregnant women. ${ }^{31}$

The HPs training followed the ABCD approach for brief behavioural counselling for Aboriginal pregnant women (Ask about smoking; Brief advice to quit; provide Cessation support and Discuss the psychosocial context of smoking), together with an expedited offer of NRT if a woman is unable to quit without medication. ${ }^{38}$ The training included specific guidance and resources focusing on improving knowledge and skills for recommending NRT during pregnancy. ${ }^{29} 3435$

Development of the intervention was based on formative research, ${ }^{13} 1822$ 39-42 and extensive consultation with Aboriginal stakeholders and communities. ${ }^{30}{ }^{35}$ Development was also informed by a rigorous theoretical analysis, ${ }^{34}$ based on the Behaviour Change Wheel ${ }^{43}$ and the linked Theoretical Domains Framework (TDF). ${ }^{44}$ The Behaviour Change Wheel is a parsimonious model developed from an overarching synthesis of behaviour intervention frameworks. ${ }^{43}$ The TDF is a validated and integrative theoretical framework developed for behaviour change research and cross-disciplinary implementation. ${ }^{44} 45$ The TDF covers a range of domains known to be relevant to professional behaviour change and has been applied across a wide range of clinical situations. ${ }^{45}$ Both the Behaviour Change Wheel and the TDF are used to practically identify and remediate barriers to achieving evidence-based care.

\section{Survey}

A 102-item self-administered online or hard copy survey. Complete description of the survey measures can be found in online supplementary file $1 .^{29}$

The items reported in this paper are defined as follows:

\section{SCC knowledge}

SCC knowledge was measured with a total composite score of 24 true/false statements. Of these, 12 statements were related to NRT use in pregnancy, so two additional separate knowledge composite scores were calculatedone specific to NRT (12 statements) and one general knowledge composite score (12 statements).

\section{Attitudes to providing SCC}

Attitudes to providing SCC were measured using fivepoint Likert scales (Strongly disagree (1) to Strongly agree (5); or Not often (1) to Very often (5)) for 15 statements covering 13 domains from the $\mathrm{TDF}^{45}$ (knowledge, reinforcement, role/identity, beliefs about capabilities, optimism, beliefs about consequences, social influence/ subjective norm, goals/priority, memory/attention, environmental context and resources, emotions/stress, intentions and behavioural regulation). The Likert scales were dichotomised to 'Agree/Strongly agree' versus rest; or 'Not often (1)/(2) versus rest. A mean composite score was created for all of the TDF domains. Seven domains from the TDF included an additional separate question regarding the prescription or recommendation of NRT (knowledge, role/identity, beliefs about capabilities, optimism, intentions, goals/priority and memory/attention); measured and dichotomised as above. A mean composite score for all NRT-specific TDF statements was also created.

In addition, self-reported perceptions of using NRT in pregnancy were measured, rating the safety for the fetus (dichotomised to 'Very safe/Always safer than smoking' vs else), effectiveness in NRT as an aid to pregnant smokers to quit (dichotomised to 'Very/Moderately effective vs 'Low' effectiveness) and perceived adherence of pregnant smokers' use of NRT (dichotomised to 'Most adhere well' vs Equal numbers adhere well and poorly/ Few adhere well).

\section{Provision of SCC}

Provision of SCC was measured using five-point Likert scales (Never to Always) on 12 different SCC components (online supplementary file 1). The Likert scales were dichotomised into 'Often/Always' versus rest. NRT prescription was also measured in proportion reporting 'Never' prescribing NRT (dichotomised to 'Never' vs else). HPs assessment of exposure to other substances that include nicotine (such as electronic cigarette or secondhand smoke, see online supplementary file 1) was measured and dichotomised as above.

\section{Usefulness of educational resources}

Usefulness of educational resources was measured using five-point Likert scales (Not useful at all to Very useful) for each webinar session and for each educational resource.

\section{Sample size}

Sample size calculations for this study were guided by the primary outcome of pregnant women's recruitment rate, ${ }^{29}$ assuming that for each service the expected recruitment would be 10 eligible consenting women. This resulted in six services participating, with an expected 5-10 HPs eligible at each service (total 30-60 HPs). ${ }^{29}$

\section{Analysis}

Demographic and patient or practice information is presented as means (SD) or counts (\%). Changes between the time points were examined using mixed modelling to account for repeated measures on some of the HPs; a fixed effect for time period was included, and a random effect for HP and service were modelled. A random intercept for the cluster was not modelled due to 
the low number of clusters (three). Outcomes with Likert scale answers were originally modelled using ordinal logistic mixed modelling; however, due to low numbers, none of the models converged. Therefore, responses were dichotomised and logistic mixed modelling was used. Results are presented for each response, and the OR for the dichotomised outcome (with $95 \%$ CI; post compared with pre) was calculated. Mean composite scores were modelled using linear mixed modelling; modelling was performed as above; however, the random intercept for service was always 0 , and was removed from linear mixed modelling; crude mean scores for each time point and differences with 95\% CI (post compared with pre) are presented. Model residuals were checked for heterogeneity and normality; empirical standard errors were calculated to account for any minor assumption violations.

Intraclass correlation coefficient (ICC) for HPs correlation between time points, and between-service correlation were calculated.

\section{Sensitivity analysis of paired HPs responses}

Sensitivity analysis of paired HPs responses was performed using responses from HPs who completed both presurveys and postsurveys; Wilcoxon signed-rank test was used to compare ordinal responses, and paired t-test to compare composite means between the two time points.

Statistical analyses were programmed using SAS V.9.4. A priori, $\mathrm{p}<0.05$ (two-tailed) was used to indicate statistical significance.

\section{Registration}

The study was registered with the Australian and New Zealand Clinical Trials Registry.

\section{Patient (HPs) involvement}

The study was designed in collaboration with two AMSs, who also participated in the study. ${ }^{30}$ A working party that consisted of various HPs (and female community members) met with the researchers to collaboratively develop the educational resource package, which was also tested through focus groups of HPs from three other AMSs participating in the study. ${ }^{35}$ Each service employed one HPs (either an Aboriginal Health Worker or a midwife) to act as the research facilitator. They were in charge of recruiting HPs and conduct of the study. At the end of the study, members of the research team visited each service and discussed study results. An infographic showing study results was sent to all services.

\section{RESULTS}

Of 93 eligible HPs in the six services, 50 (54\%) consented and filled out at least one survey-45 (90\%) completed the pretraining survey and $20(40 \%)$ completed the post-training survey. Fifteen (30\%) HPs completed both surveys (table 1). Overall, 39 (42\%) HPs participated in the webinar training. Figure 2 shows the breakdown of HPs for each service according to eligibility, training attendance and survey completion. HPs recruitment rate at the different AMSs ranged from 33\% to $72 \%$. All types of HPs were recruited, including 17 general practitioners, 17 nurses/midwives, 10 Aboriginal Health Workers and six other (eg, social worker). One service (\#6) did not provide any post-training surveys. Postsurveys were completed between 1 and 6 months postintervention (range 29-182 days, mean 97.5 days).

\section{SCC knowledge}

Total knowledge mean composite score improved significantly from pre to post $(77.9 \%$ vs $84 \%$ correct, difference $5.95,95 \%$ CI 1.57 to 10.32 , ICC HPs 0.76 ). Breaking the total knowledge composite score into an NRT-specific and a non-NRT-specific mean composite scores shows a significant improvement for NRT-specific score but not for the non-NRT-specific score $(78.8 \%$ vs $68.2 \%$ correct, difference $9.9,95 \%$ CI 3.66 to 16.14 , ICC HPs $0.71 ; 89.2 \%$ vs $87.7 \%$, difference $1.89,95 \%$ CI -3.46 to 7.24 , ICC HPs 0.52 , respectively).

\section{Attitudes to providing SCC}

Table 2 provides crude responses and logistic modelling OR for all TDF domains. Seventy-five per cent of HPs reported 'Strongly agreeing/Agreeing' to having sufficient resources postintervention compared with $36.4 \%$ preintervention (OR 5.70, 95\% CI 1.44 to 22.58). Optimism for effectiveness of their intervention with pregnant women who smoked showed a non-significant, but substantial effect size for improvement (60\% post vs $31.8 \%$ pre, OR $3.3,95 \%$ CI 0.95 to 11.51$)$. Similarly, a non-significant, but substantial effect size was seen for reporting 'Not often' forgetting to provide counselling ( $75 \%$ post vs $59.1 \%$ pre, OR $2.21,95 \%$ CI 0.55 to 8.87 ). Five out of the seven TDF domains NRT-specific questions also showed a non-significant, but substantial effect size (knowledge about how to counsel on NRT, confidence in ability to recommend/prescribe NRT, optimism that NRT will be effective, intention to prescribe NRT and less often forgetting to recommend/prescribe NRT (table 2)).

The total mean composite score for the general TDF domains improved significantly from 3.65 (SD 0.4) to 3.87 (SD 0.4) (difference $0.23,95 \%$ CI 0.05 to 0.41 , ICC HPs 0.56). Mean composite NRT-specific TDF score was also significantly improved from 3.37 (0.6) to $3.64(0.7)$ (difference $0.36,95 \%$ CI 0.13 to 0.6 , ICC HPs 0.74 ).

Table 3 presents the responses for self-reported perceptions on NRT safety, effectiveness and adherence, all showing a non-significant but substantial effect size for improvement.

\section{Provision of SCC}

Provision of SCC is shown in figure 3 and online supplementary file 3 . The odds of reporting on the provision of the different SCG components 'Often/Always' did not change significantly preintervention to postintervention, with the exception of 'Recording smoking status' in 
Table 1 Demographic characteristics of participants $(n=50), n(\%)$

\begin{tabular}{|c|c|c|c|}
\hline Characteristic & Total $n=50$ & Pre-training $n=45$ & Post-training $n=20$ \\
\hline Age (mean, range), years & $43.8(18-64)$ & $43.7(18-64)$ & $45.6(29-64)$ \\
\hline Gender-female & $43(86 \%)$ & $39(86.7 \%)$ & $18(90 \%)$ \\
\hline \multicolumn{4}{|l|}{ Smoking status } \\
\hline Current smoker (daily and occasional) & $5(10 \%)$ & $5(11.1 \%)$ & $2(10 \%)$ \\
\hline Ex-smoker & $17(34 \%)$ & $13(28.9 \%)$ & $10(50 \%)$ \\
\hline Never smoker & $28(56 \%)$ & $27(60 \%)$ & $8(40 \%)$ \\
\hline \multicolumn{4}{|l|}{ Profession } \\
\hline General practitioner & $17(34 \%)$ & $15(33.3 \%)$ & $4(20 \%)$ \\
\hline Midwife/nurse & $16(32 \%)$ & $16(35.6 \%)$ & $6(30 \%)$ \\
\hline Aboriginal Health Worker & $10(20 \%)$ & $9(20 \%)$ & $5(25 \%)$ \\
\hline Other* & $7(14 \%)$ & $5(11.1 \%)$ & $5(25 \%)$ \\
\hline \multicolumn{4}{|l|}{ Work experience } \\
\hline Less than 10 years & $24(48 \%)$ & $20(44.4 \%)$ & $13(65 \%)$ \\
\hline 10-19years & $10(20 \%)$ & $10(22.2 \%)$ & $1(5 \%)$ \\
\hline 20 or more years & $16(32 \%)$ & $15(33.3 \%)$ & $6(30 \%)$ \\
\hline \multicolumn{4}{|l|}{ Service } \\
\hline 1 & $4(6 \%)$ (one matched) & $3(6.7 \%)$ & $2(10 \%)$ \\
\hline 2 & $3(6 \%)$ (three matched) & $3(6.7 \%)$ & $3(15 \%)$ \\
\hline 3 & 13 (26\%) (four matched) & $11(24.4 \%)$ & $6(30 \%)$ \\
\hline 4 & 7 (14\%) (three matched) & $6(13.3 \%)$ & $4(20 \%)$ \\
\hline 5 & 13 (26\%) (four matched) & $12(26.7 \%)$ & $5(25 \%)$ \\
\hline 6 & 10 (20\%) (0 matched) & $10(22.2 \%)$ & $0(0 \%)$ \\
\hline
\end{tabular}

*Other professions included all other allied health professionals, including family strengthening worker, social worker and psychologist.

the medical file, which was significantly reduced $(96 \%$ vs $75 \%$ for pre and post, respectively, OR $0.14,95 \% \mathrm{CI}$ 0.02 to 0.99$)$. 'Advise to quit smoking', 'Assess nicotine dependence' and 'Assist (provide cessation support)' showed a non-significant, but substantial effect size for improvement. No change was observed for self-report on NRT prescribing rate (neither an increase in reporting 'Often/Always' prescribing, nor a decrease in 'Never' prescribing).

The odds of reporting 'Often/Always' assessing the use of cannabis were unchanged pretraining to posttraining (OR $0.90,95 \% \mathrm{CI} 0.21$ to 3.93 ). Assessing all other substances that expose the fetus to nicotine showed a non-significant, but substantial effect size for improvement, including the use of electronic cigarettes with nicotine (OR 2.09, 95\% CI 0.43 to 10.17), chewing tobacco (OR 2.67, 95\% CI 0.55 to 13.01) and secondhand smoke (OR 2.51, 95\% CI 0.68 to 9.31 ).

\section{Usefulness of educational resources $(n=20$, post-follow-up only)}

All of the resources except the mouse pad were rated as useful with a mean ranging from 3.89 (SD 1.1) for the patient flipchart to 4.17 (SD 0.9) for the different types of NRT poster (median 'useful (4)' and mode 'very useful (5)' for all). The mouse pad was rated as somewhat useful, with a mean of 3.22 (SD 1.2) (both median and mode 'somewhat useful'). The webinar sessions were also rated as useful with a mean of 3.6 (SD 0.9), 3.8 (SD 1.0) and 3.9 (SD 1.0) for session 1, 2, and 3, respectively (with both median and mode 'useful', except for session 3 (dedicated to NRT), which had a mode of 'very useful'). Eighty per cent $(n=16)$ of participants reported reading at least part of the treatment manual, but only four reported reading all of the manual from front to back.

\section{Subanalysis of paired HPs responses}

Similar results were seen for the paired analysis, except for assessment of other substance use that did not show any improvement in the paired analysis (online supplementary file 4). As in the non-paired analysis, both mean knowledge composite score (post 5.8\% higher than pre, $95 \%$ CI 1.32 to 10.35 ) and NRT-specific knowledge composite score (post $9.4 \%$ higher than pre, $95 \% \mathrm{CI}$ 2.72 to 16.17 ) improved significantly, and the total mean composite score for the general TDF domains improved significantly from 3.65 (SD 0.4) to 3.91 (SD 0.4) (difference $0.26,95 \%$ CI 0.04 to 0.47 ). Mean composite NRT-specific TDF score was also significantly improved from $3.4(0.5)$ to $3.84(0.5)$ (difference $0.44,95 \%$ CI 0.2 to 0.67 ). Significant improvements were seen in self-reported attitudes including optimism, knowledge on NRT, perception that 
Cluster 1

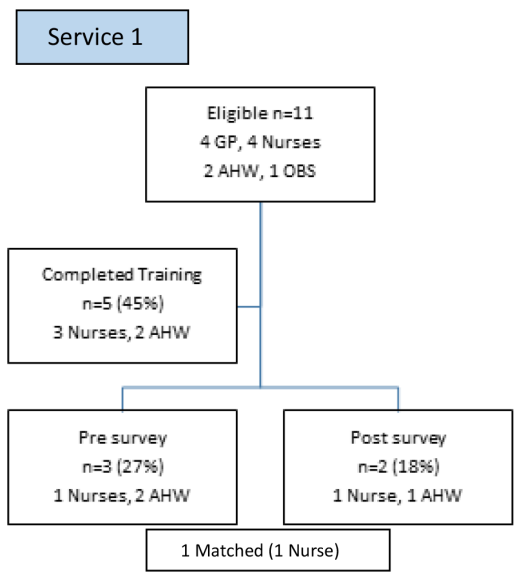

Cluster 2

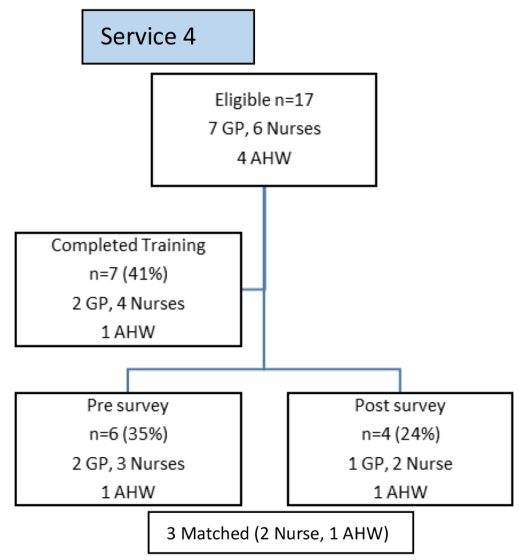

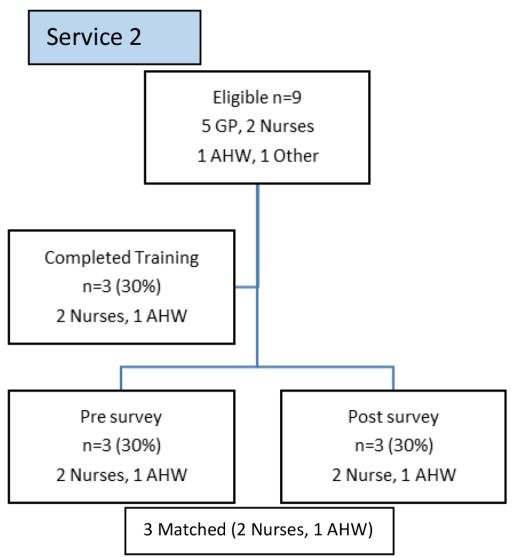

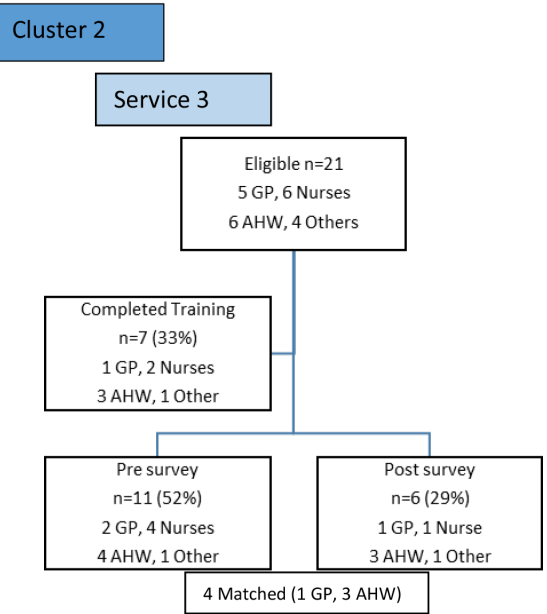

Cluster 3

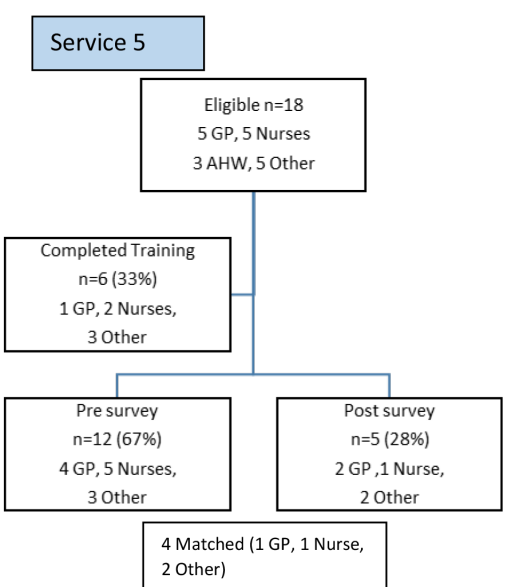

Figure 2 Health providers' eligibility, recruitment and retention per service. AHW, Aboriginal Health Worker; GP, general practitioner; OBS, Obstetrician.

recommending/prescribing NRT is part of HPs work and intention to prescribe NRT. 'Assessing' nicotine dependence also showed a significant improvement.

\section{DISCUSSION}

\section{Principal results}

'ICAN QUIT in Pregnancy' was a pilot multicomponent intervention, including live interactive webinar training. Preliminary underpowered findings showed an improvement in HPs knowledge and attitudes towards providing SCC in pregnancy. Furthermore, a non-significant, but substantial effect size was observed for improvement in several practices ('Advise', 'Assess' and 'Assist'), and in assessment of overall exposures to tobacco and nicotine, including secondhand smoke and electronic cigarette use. NRT-specific knowledge and attitudes also improved, although no change was seen in self-reported NRT recommendation/prescription rates.

\section{Limitations and strengths}

This study was a pilot study not powered to test effectiveness of changes in HPs knowledge, attitudes and practices. Our findings are suggestive that the intervention might improve knowledge, attitudes and some practices. However, multiple comparisons were performed, which might indicate that not all findings are correct. Due to the small sample size, effect sizes should be interpreted cautiously. More conclusive results will require a larger trial. These results inform the design of SISTAQUIT, which will include 30 AMSs nationally in Australia, and will be powered to test intervention effectiveness, inclusive of smoking cessation as an outcome for Aboriginal pregnant women. A major limitation of the current study was the low retention rates of the HPs, resulting in only $40 \%$ providing a post-training survey, and only one follow-up survey (instead of the planned two). The variability around when the postsurvey was completed is another limitation, as changes in knowledge, attitudes and practices may change over time. The low retention rates and small sample size limits our ability to interpret the findings, and to generalise them to all HPs and services, while raising the question of a selection bias. Assuming those who chose to answer the post-training survey might be the ones that benefited most from the intervention, 


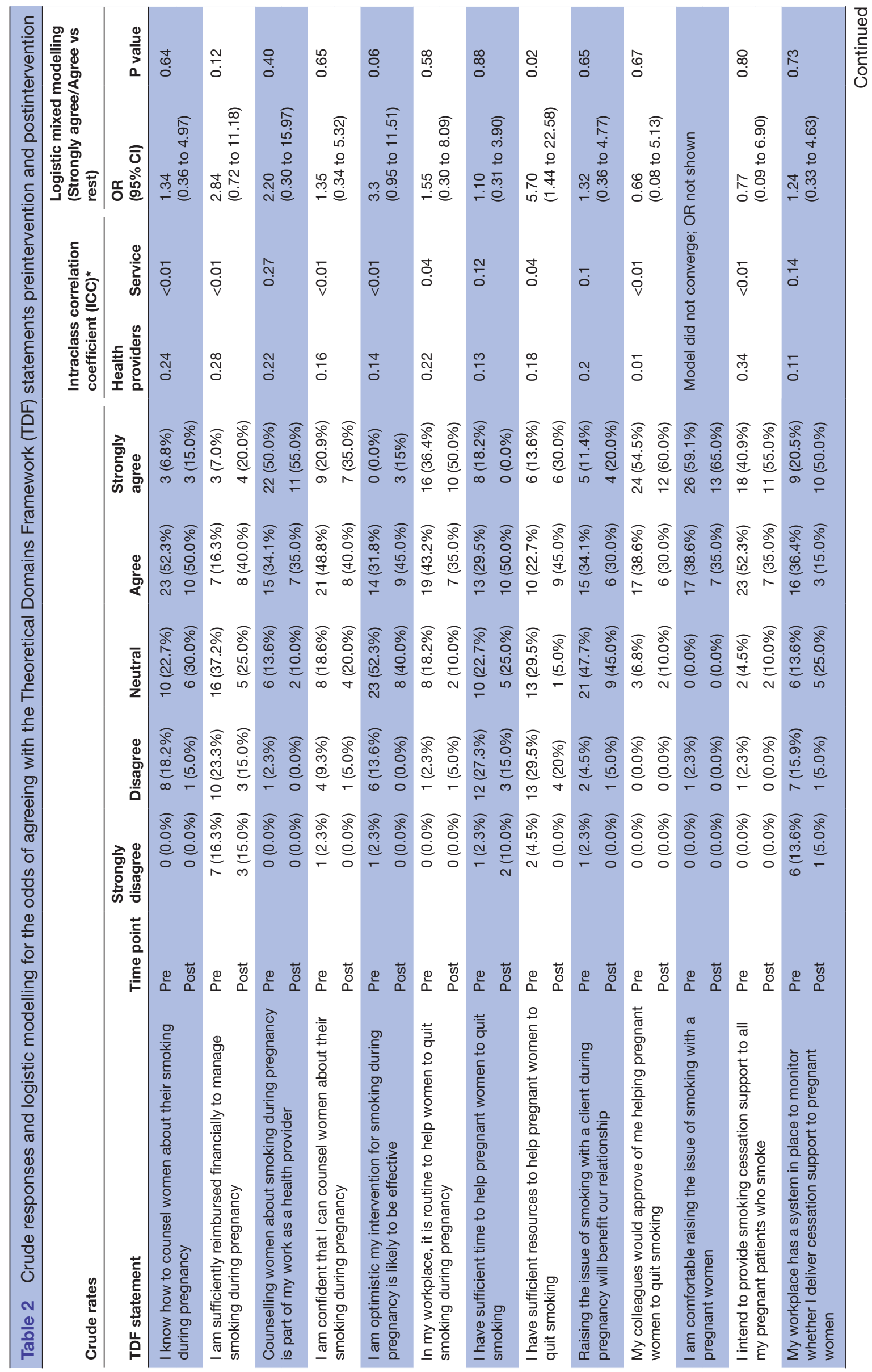




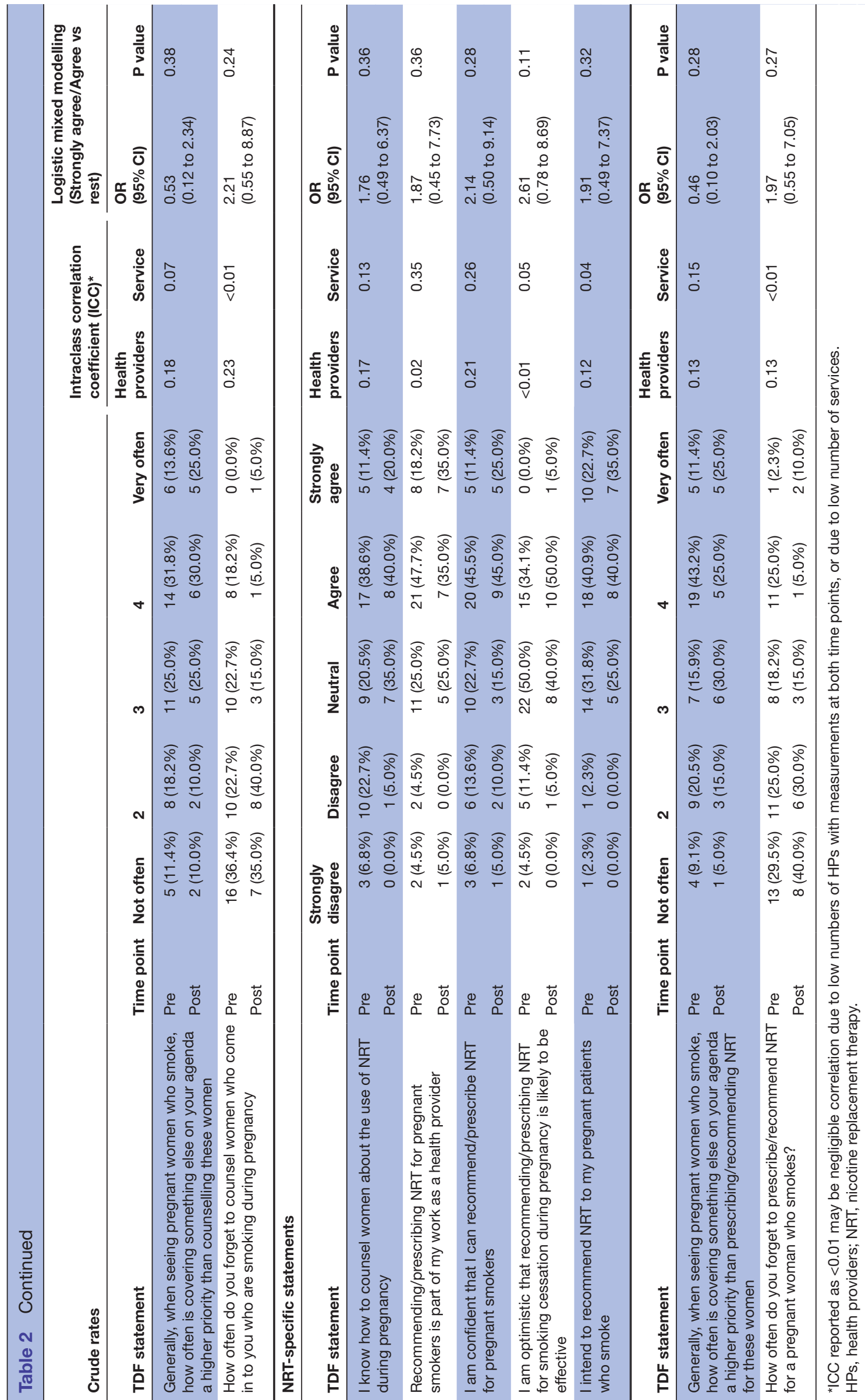


actual changes in HP attributes may, in reality, be lower than results reported. Participating HPs might have been those who had more interest in smoking cessation; thus, representing better performing HPs. Despite this, to the best of our knowledge, this is the most extensive study to date undertaken within Australian AMSs evaluating smoking cessation during pregnancy. Inclusion of six services, from three different states, aids the potential generalisability of these findings.

\section{Comparison with prior work}

A recent systematic review identified 14 interventions on smoking cessation during pregnancy, which included data on HPs. ${ }^{28}$ This review found that interventions had a modest positive impact on the various SCC components. A few interventions also included data regarding knowledge and attitudes of HPs, ${ }^{46-50}$ also showing an overall positive increase. None of the interventions included in this review were tailored to HPs treatment in indigenous populations, although a few were conducted with HP treating low socioeconomic populations. ${ }^{46} 48$ 51-53 Our results are similar and provide further evidence that interventions can modestly increase HPs knowledge, attitudes and practices in supporting pregnant women to quit smoking, including in an Aboriginal context.

None of the interventions included in this systematic review incorporated any components specifically addressing NRT prescription, and/or knowledge and attitudes regarding NRT prescription, with only one measuring changes in NRT prescription rates preintervention and postintervention, with no differences observed. ${ }^{54}$ Our intervention was designed specifically to address low NRT prescription rates, and is the first to include specific measures on knowledge, attitudes and practices of recommending/prescribing NRT during pregnancy.

None of the previous interventions focusing on HPs care during pregnancy used webinar as the training delivery method. ${ }^{28}$ Lack of time, limited funding and lack of training are consistent barriers identified for health services and HPs to provide SCC during pregnancy. ${ }^{20255}$ Webinar is a novel approach and, if found to be effective in the SISTAQUIT trial, might provide an effective measure to conduct training and skill development, reducing both costs and time for travel. Thus, webinar training may be suitable specifically within rural and remote medical services. To the best of our knowledge, this study was the first to use this training mode for improving SCC during pregnancy.

\section{Implication for policy and future research}

As part of this intervention, oral NRT was supplied free within the service directly to the women throughout the study period. NRT patches were to be supplied as usual by providing women with a prescription, which they would fill at a pharmacy for a subsidised governmental rate. An analysis of actual provision of oral $\mathrm{NRT}^{31}$ throughout the study showed that $55 \%(12 / 22)$ of recruited women accepted an oral NRT offer, with $6 / 12$ reporting using it 


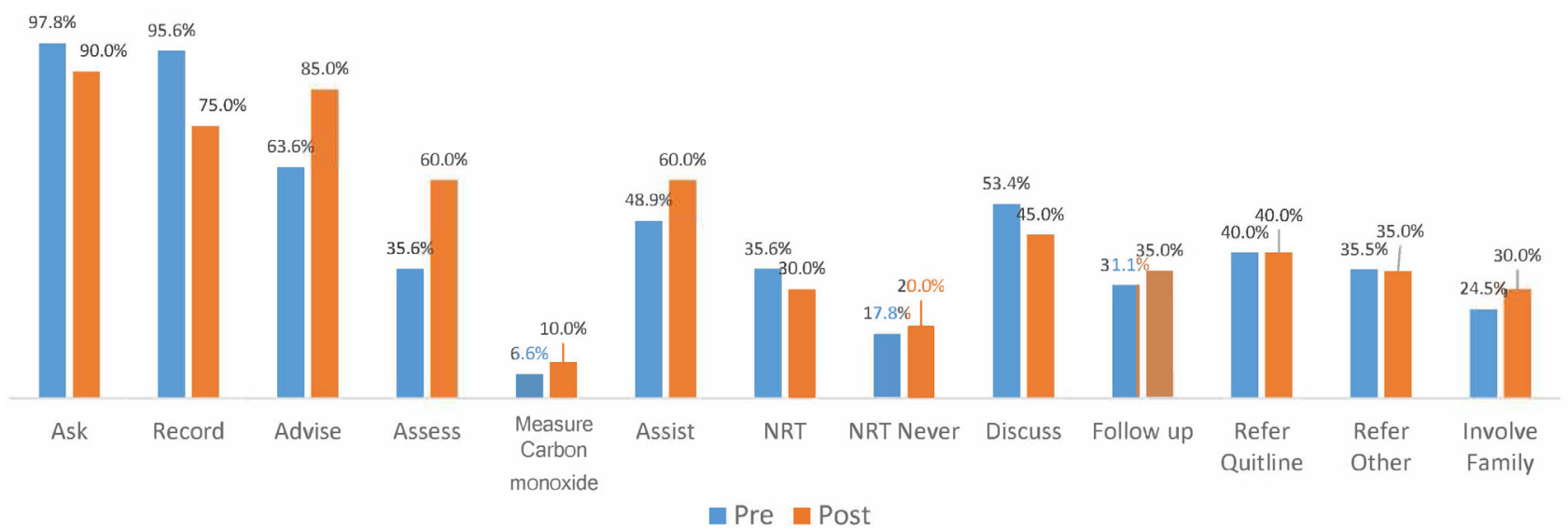

Figure 3 Proportion of health providers self-reporting provision of SCC components 'Often/Always' vs else (and for NRT prescription 'Never' vs else). NRT, nicotine replacement therapy; SCC, smoking cessation care.

at least occasionally. The data on NRT acceptance and usage might suggest that although HPs completing the postsurvey did not report changing NRT practices, their improved NRT-related knowledge and attitudes might have contributed to women's perception and NRT acceptance levels. Computerised data from four services showed that government prescriptions for the NRT patch did not change across three services, and improved in one service (from one woman offered a prescription pretraining to four women post-training). Having the oral NRT within the service, supplied 'on the spot' free of charge, may have had an impact on both HPs and women's practices, not reflected by the postsurvey of HPs. Reducing cost and logistic barriers may be helpful in improving the use of NRT in pregnancy.

Assessment of overall nicotine exposure of the fetus is important as nicotine in itself may be harmful, especially to the lung and brain development. ${ }^{56}{ }^{57}$ Previous research has shown that over $75 \%$ of Australian HPs ask about tobacco use during pregnancy, ${ }^{2258}$ yet the proportion asking about exposure to other nicotine-containing products is limited $(9 \%-38 \%){ }^{59}$ Raising awareness of these exposures by training is important with the emergence of new nicotine-containing products, such as electronic cigarettes, and the uncertainties around the harm versus benefit of these alternate products in pregnancy. HPs should advise women that the best health benefits are seen with complete elimination of nicotine exposure in all forms. Future research needs to address whether, similar to NRT, ${ }^{1617}$ electronic cigarettes can offer a 'harm reduction' strategy for pregnant women as well.

We were unable to provide the audit and feedback component at a service level as planned. ${ }^{31}$ Audit and feedback have been found to be an important component in interventions aimed at changing HPs behaviour in general. ${ }^{60}$ Future interventions should find ways to incorporate this as a feasible and acceptable method.

\section{Changes to the future SISTAQUIT design}

Results from this study have informed intervention development for the SISTAQUIT trial, including further incentives for HPs to participate (such as category one continuing professional development points and a prize-draw for survey completion). Surveys will be shortened, and training case study discussions will include videoed examples featuring the $\mathrm{ABCD}$ methods and how to conduct discussions about NRT with the pregnant woman. A full description of the recommended changes is described elsewhere. ${ }^{31}$

The 'ICAN QUIT in Pregnancy' pilot intervention was feasible with promising preliminary results. Strategies to increase HPs provision of NRT need to be improved, and the webinar training has been refined for the larger SISTAQUIT study. However, even a small increase in smoking cessation rates can have significant positive health impacts, both for the mother and baby, and help to close the health and life expectancy gap between the Aboriginal and non-Aboriginal Australian population.

\section{CONCLUSIONS}

Training HPs through live interactive webinar training and provision of educational resources was feasible, and might have a positive impact on HPs provision of SCC to pregnant Aboriginal women. Changes in NRT prescription rates may require additional intensive measures. The AMSs have a significant role in the Aboriginal community; therefore, improving capacity to support pregnant Aboriginal women to quit is vital and should be part of a comprehensive approach to tackle Aboriginal smoking rates during pregnancy. Using webinar training may have the potential to save both time and funds; thus, suitable especially for rural and remote medical services.

\section{Author affiliations}

${ }^{1}$ School of Medicine and Public Health, University of Newcastle, Callaghan, New South Wales, Australia

${ }^{2}$ Wollotuka Institute, University of Newcastle, Callaghan, New South Wales, Australia ${ }^{3}$ Clinical Research Design, Information Technology and Statistical Support (CReDITSS) Unit, Hunter Medical Research Institute, Newcastle, New South Wales, Australia

${ }^{4}$ UCL Centre for Behaviour Change, University College London, London, UK 
${ }^{5}$ School of Medicine, Western Sydney University, Penrith South DC, New South Wales, Australia

Acknowledgements The authors thank the Aboriginal communities who have contributed to the development and implementation of the 'ICAN QUIT in Pregnancy' intervention. This includes staff and patients of the Biripi Aboriginal Corporation, the Tobwabba Aboriginal Medical Service, the Pangula Mannamurna Aboriginal Corporation, the Riverina Medical and Dental Aboriginal Corporation, the Wuchopperen Health Service Ltd and the Tharawal Aboriginal Corporation. The authors also thank the members of the Stakeholder and Consumer Aboriginal Advisory Panel for 'ICAN QUIT in Pregnancy' for the ongoing guidance and support in implementing the study. We would also like to acknowledge Lauren Pollack who worked as a research assistant throughout the study and was responsible for the everyday administration of running this study.

Collaborators The following collaborators are in the 'ICAN QUIT in Pregnancy' Pilot Group: GSG, BB, Peter O'Mara, Marilyn Clarke, Chris Oldmeadow, Alan Clough, Kristin Carson, JR, YBZ, MB, Katherine Boydell, Ling Li Lim, MG, Roger Smith, Yvonne Cadet-James, Renee Bittoun, Lou Atkin, Brett Cowling and Lisa Orcher.

Contributors GSG, YBZ and MB conceived and designed the study. YBZ led the data collection and analysis plan, and wrote the manuscript. $M B$ and $M G$ advised on Aboriginal community consultations and adherence to ethical guidelines to research with Aboriginal communities. BB and JR advised on methodology and implementation of the research. KP performed the statistical analysis, and $\mathrm{CO}$ oversaw the analysis and advised on methodology. LA advised on the design and the analysis using the Theoretical Domains Framework and Behaviour Change Wheel. The 'ICAN QUIT in Pregnancy' Pilot Group advised on the research design and implementation. GSG oversaw the entire study. All co-authors critically reviewed and approved the final manuscript.

Funding This work was supported by the NSW Ministry of Health and the Hunter Cancer Research Alliance. YBZ is supported by the University of Newcastle and Hunter Cancer Research Alliance PhD Scholarship. MB is supported by the University of Newcastle and Australian Heart Foundation Indigenous Scholarship (\#101555). GSG is supported by the National Health and Medical Research Council and the Cancer Institute New South Wales Early Career Research Fellowships (APP1092085 and 15ECF/I-52).

Competing interests YBZ has received funds in the past (2012-2015) from the Novartis NCH, who used to distribute NRT in Israel. She has not received any funding from pharmaceutical companies in Australia.

Patient consent for publication Not required.

Ethics approval Approval for this study was granted by the University of Newcastle Human Research Ethics Committee (HREC) (REF \#H-2015-0438), the Aboriginal Health \& Medical Research Council (AH\&MRC) HREC (REF \#1140/15), the South Australia Aboriginal HREC (REF \#04-16-652) and the Far North Queensland HREC (REF \#16/QCH/34-1040).

Provenance and peer review Not commissioned; externally peer reviewed.

Data sharing statement № additional unpublished data are available.

Open access This is an open access article distributed in accordance with the Creative Commons Attribution Non Commercial (CC BY-NC 4.0) license, which permits others to distribute, remix, adapt, build upon this work non-commercially, and license their derivative works on different terms, provided the original work is properly cited, appropriate credit is given, any changes made indicated, and the use is non-commercial. See: http://creativecommons.org/licenses/by-nc/4.0/.

\section{REFERENCES}

1. Chamberlain C, O'Mara-Eves A, Porter J, et al. Psychosocial interventions for supporting women to stop smoking in pregnancy. Cochrane Database Syst Rev 2017;2:CD001055.

2. Lange S, Probst C, Rehm J, et al. National, regional, and global prevalence of smoking during pregnancy in the general population: a systematic review and meta-analysis. Lancet Glob Health 2018;6:e769-e776.

3. Australian Institute of Health and Welfare. Australia's mothers and babies 2015-in brief. Canberra 2017.

4. Australian Institute of Health and Welfare. Australia's welfare 2017: in brief. Canberra.

5. Brady M. Historical and cultural roots of tobacco use among Aboriginal and Torres Strait Islander people. Aust N Z J Public Health 2002;26:120-4.
6. Australian bureau of statistics. National aboriginal and torres strait islander social survey, 2014-15.

7. van der Sterren A, Greenhalgh EM, Knoche D, et al. 8.1 Aboriginal peoples and Torres Strait Islanders: social disadvantage, health and smoking - an overview. In Scollo, MM and Winstanley, MH [editors]. Tobacco in Australia: Facts and issues. Cancer Council Victoria. 2016 http://www.tobaccoinaustralia.org.au/chapter-8-aptsi/8-1-overview.

8. Read P, Japaljarri E. The price of tobacco: the journey of the Warlmala to Wave Hill, 1928. Aboriginal History 1978;2:140-8.

9. Al-Yaman F. The australian burden of disease study: impact and causes of illness and death in aboriginal and torres strait islander people, 2011. Public Health Res Pract 2017;27.

10. Australia C. Department of the prime minister and cabinet: Closing the Gap Prime Minister's Report, 2018.

11. Gould GS, Munn J, Watters T, et al. Knowledge and views about maternal tobacco smoking and barriers for cessation in Aboriginal and Torres Strait Islanders: A systematic review and metaethnography. Nicotine Tob Res 2013;15:863-74.

12. Gould GS, Munn J, Avuri S, et al. "Nobody smokes in the house if there's a new baby in it": Aboriginal perspectives on tobacco smoking in pregnancy and in the household in regional NSW Australia. Women Birth 2013;26:246-53.

13. Bovill M, Gruppetta M, Cadet-James Y, et al. Wula (Voices) of Aboriginal women on barriers to accepting smoking cessation support during pregnancy: Findings from a qualitative study. Women Birth 2018;31:10-16.

14. Twyman L, Bonevski B, Paul C, et al. Perceived barriers to smoking cessation in selected vulnerable groups: a systematic review of the qualitative and quantitative literature. BMJ Open 2014;4:e006414.

15. Gould GS, Watt K, McEwen A, et al. Predictors of intentions to quit smoking in Aboriginal tobacco smokers of reproductive age in regional New South Wales (NSW), Australia: quantitative and qualitative findings of a cross-sectional survey. BMJ Open 2015;5:e007020.

16. Coleman T, Chamberlain C, Davey MA, et al. Pharmacological interventions for promoting smoking cessation during pregnancy. Cochrane Database Syst Rev 2015;12:CD010078.

17. Bar-Zeev Y, Lim Ling L, Gruppeta M, et al. Nicotine replacement therapy for smoking cessation in pregnancy - a narrative review. Medical Journal of Australia 2018;208.

18. Bar-Zeev Y, Bonevski B, Gruppetta M, et al. Clinician factors associated with prescribing nicotine replacement therapy in pregnancy: A cross-sectional survey of Australian obstetricians and general practitioners. Aust N Z J Obstet Gynaecol 2018;58:366-70.

19. Gould GS, Bar-Zeev Y, Twyman L, et al. Health Professionals Performing the " $5 \mathrm{As}$ " for smoking cessation and prescribing nicotine replacement therapy during pregnancy: meta-analysis of a systematic review. 2017 Hunter Cancer Research Alliance Symposium "Translating research for impact". Asia-Pacific Journal of Clinical Oncology 2017;13:16.

20. Okoli CTC, Greaves L, Bottorff JL, et al. Health care providers' engagement in smoking cessation with pregnant smokers. J Obstet Gynecol Neonatal Nurs 2010;39:64-77.

21. Baxter S, Everson-Hock E, Messina J, et al. Factors relating to the uptake of interventions for smoking cessation among pregnant women: a systematic review and qualitative synthesis. Nicotine Tob Res 2010;12:685-94.

22. Zeev YB, Bonevski B, Twyman L, et al. Opportunities missed: a cross-sectional survey of the provision of smoking cessation care to pregnant women by australian general practitioners and obstetricians. Nicotine Tob Res 2017;19:636-41.

23. Tzelepis F, Daly J, Dowe S, et al. Supporting aboriginal women to quit smoking: antenatal and postnatal care providers' confidence, attitudes, and practices. Nicotine Tob Res 2017;19:642-6.

24. Eades SJ, Sanson-Fisher RW, Wenitong M, et al. An intensive smoking intervention for pregnant Aboriginal and Torres Strait Islander women: a randomised controlled trial. Med J Aust 2012;197:42-6.

25. Passey M, Stirling J. Evaluation of 'Stop Smoking in its Tracks': an intensive smoking cessation program for pregnant Aboriginal women incorporating contingency-based financial rewards. Public Health Res Pract 2018;28:e28011804.

26. Hudmon KS, Hoch MA, Vitale FM, et al. Tobacco cessation education for pharmacists: Face-to-face presentations versus live webinars. $J$ Am Pharm Assoc 2014;54:42-4.

27. Sarna L, Bialous SA, Ong MK, et al. Increasing nursing referral to telephone quitlines for smoking cessation using a web-based program. Nurs Res 2012;61:433-40.

28. Bar-Zeev Y, Bonevski B, Lim LL, et al. Improving health providers smoking cessation care in pregnancy: a systematic review and metaanalysis. Addict Behav 2019;93:29-38. 
29. Bar-Zeev Y, Bonevski B, Bovill M, et al. The Indigenous Counselling and Nicotine (ICAN) QUIT in Pregnancy Pilot Study protocol: a feasibility step-wedge cluster randomised trial to improve health providers' management of smoking during pregnancy. BMJ Open 2017;7:e016095.

30. Bovill M, Bar-Zeev Y, Gruppetta M, et al. Collective and negotiated design for a clinical trial addressing smoking cessation supports for Aboriginal and Torres Strait Islander mothers in NSW, SA and Qld developing a pilot study. Aust J Prim Health 2017;23:497-503.

31. Gould GS, Bovill M, Pollock L, et al. Feasibility and acceptability of Indigenous Counselling and Nicotine (ICAN) QUIT in Pregnancy multicomponent implementation intervention and study design for Australian Indigenous pregnant women: a pilot cluster randomised step-wedge trial. Addict Behav 2019;90:176-90.

32. Australian Institute of Health and Welfare. Healthy futures-aboriginal community controlled health services: report card. Canberra, 2016.

33. NSW Health. Workforce planning and development. Definition of an aboriginal health worker. Report \#IB2018 018.

34. Gould GS, Bar-Zeev Y, Bovill M, et al. Designing an implementation intervention with the Behaviour Change Wheel for health provider smoking cessation care for Australian Indigenous pregnant women. Implement Sci 2017;12:114.

35. Bar-Zeev Y, Bovill M, Bonevski B, et al. Assessing and validating an educational resource package for health professionals to improve smoking cessation care in aboriginal and torres strait islander pregnant women. Int J Environ Res Public Health 2017;14:1148.

36. Dempsey D, Jacob P, Benowitz NL. Accelerated metabolism of nicotine and cotinine in pregnant smokers. $J$ Pharmacol Exp Ther 2002;301:594-8.

37. The Australian Goverment Depertment of Health. The pharmacutical benefits scheme: listings on the PBS for Aboriginal and torres strait islander people. http://www.pbs.gov.au/info/publication/factsheets/ shared/pbs-listings-for-aboriginal-and-torres-strait-islander-people.

38. Gould GS, Bittoun R, Clarke MJ. A pragmatic guide for smoking cessation counselling and the initiation of nicotine replacement therapy for pregnant aboriginal and torres strait islander smokers. $J$ Smok Cessat 2015;10:96-105.

39. Gould GS, Bovill M, Clarke MJ, et al. Chronological narratives from smoking initiation through to pregnancy of Indigenous Australian women: A qualitative study. Midwifery 2017;52:27-33.

40. Gould GS. Exploring the barriers and enablers to smoking cessation in pregnant Aboriginal and Torres Strait Islander women. Austral Epidemiol 2014;21:31-5.

41. Gould GS, Watt K, Stevenson L, et al. Developing anti-tobacco messages for Australian Aboriginal and Torres Strait Islander peoples: evidence from a national cross-sectional survey. BMC Public Health 2014;14:250.

42. Gould GS, Clarke MJ, Cadet-James Y, et al. "Our smoking and smoke-free stories" by Aboriginal women: a qualitative study. Perth, WA: GP16 Conference, 2016.

43. Michie S, van Stralen MM, West R. The behaviour change wheel: a new method for characterising and designing behaviour change interventions. Implement Sci 2011;6:1-12.

44. French SD, Green SE, O'Connor DA, et al. Developing theoryinformed behaviour change interventions to implement evidence into practice: a systematic approach using the Theoretical Domains Framework. Implement Sci 2012;7:38.

45. Cane J, O'Connor D, Michie S. Validation of the theoretical domains framework for use in behaviour change and implementation research. Implement Sci 2012;7:37.

46. Althabe F, Alemán A, Berrueta $\mathrm{M}$, et al. A multifaceted strategy to implement brief smoking cessation counseling during antenatal care in argentina and uruguay: a cluster randomized trial. Nicotine Tob Res 2016;18:1083-92.

47. Albrecht S, Kelly-Thomas K, Osborne JW, et al. The SUCCESS program for smoking cessation for pregnant women. $J$ Obstet Gynecol Neonatal Nurs 2011;40:520-31.

48. Velasquez MM, Hecht J, Quinn VP, et al. Application of motivational interviewing to prenatal smoking cessation: training and implementation issues. Tob Control 2000;9(Suppl 3):36iii-40.

49. Valanis B, Labuhn KT, Stevens NH, et al. Integrating prenatalpostnatal smoking interventions into usual care in a health maintenance organization. Health Promot Pract 2003;4:236-48.

50. Naylor PJ, Adams JS, McNeil D. Facilitating changes in perinatal smoking. The impact of a stage-based workshop for care-providers in British Columbia. Can J Public Health 2002;93:285-90.

51. Yusem SH, Rosenberg KD, Dixon-Gray L, et al. Public health nursing acceptance of the $5 \mathrm{~A}^{\prime}$ 's protocol for prenatal smoking cessation. Californian Journal of Health Promotion 2004;2:1-10.

52. Secker-Walker RH, Solomon LJ, Flynn BS, et al. Training obstetric and family practice residents to give smoking cessation advice during prenatal care. Am J Obstet Gynecol 1992;166:1356-63.

53. Moss DR, Cluss PA, Watt-Morse M, et al. Targeting pregnant and parental smokers: long-term outcomes of a practice-based intervention. Nicotine Tob Res 2009;11:278-85.

54. Chertok IR, Casey ML, Greenfield K. Approach to addressing prenatal smoking in West Virginia. W V Med J 2014;110:36-40.

55. McFarlane KA, Judd J, Wapau $\mathrm{H}$, et al. How primary health care staff working in rural and remote areas access skill development and expertise to support health promotion practice. Rural Remote Health 2018;18:4413.

56. England LJ, Aagaard K, Bloch M, et al. Developmental toxicity of nicotine: a transdisciplinary synthesis and implications for emerging tobacco products. Neurosci Biobehav Rev 2017;72:176-89.

57. Smith AM, Dwoskin LP, Pauly JR. Early exposure to nicotine during critical periods of brain development: Mechanisms and consequences. J Pediatr Biochem 2010;1:125-41.

58. Passey ME, D'Este CA, Sanson-Fisher RW. Knowledge, attitudes and other factors associated with assessment of tobacco smoking among pregnant Aboriginal women by health care providers: a crosssectional survey. BMC Public Health 2012;12:165.

59. Gould GS, Zeev YB, Tywman L, et al. Do clinicians ask pregnant women about exposures to tobacco and cannabis smoking, second-hand-smoke and E-cigarettes? An Australian National Cross-Sectional Survey. Int J Environ Res Public Health 2017;14:1585.

60. Ivers N, Jamtvedt G, Flottorp S, et al. Audit and feedback: effects on professional practice and healthcare outcomes. Cochrane Database Syst Rev 2012;6:CD000259. 